\title{
How Feasible is Direct Determination of Rare Earth Elements in Seawater by ICP-MS?
}

\author{
Olga V. KuZnetsova, Nikita V. Dushenko, and Andrei R. TimerbaeV ${ }^{\dagger}$ \\ Vernadsky Institute of Geochemistry and Analytical Chemistry, Kosygin Str. 19, 119991 \\ Moscow, Russian Federation
}

With the increasingly wide industrial use of rare earth elements (REEs), their release into marine systems makes it important to understand in what quantitates they occur and in what geochemical processes contribute, preferably using direct analytical methodology. In this study, analytical performance of high-resolution ICP-MS was assessed with regard to quantification of REEs in seawater without matrix separation and analyte preconcentration. With optimized sample dilution, precise and interference-free quantification of most of the REEs in samples taken from Kara Sea was obtained, with the accuracy from 3 to $9 \%$ (against the independently asserted values), repeatability 3$5 \%$, intermediate precision and reproducibility, averaging 4 and $11 \%$, respectively. The method was further validated by using a certified reference material for nearshore seawater. However, the limits of detection obtained (0.04-0.38 $\mathrm{ng} \mathrm{L}^{-1}$ ), while not significantly inferior to those obtained after sample enrichment, appear to be not low enough to analyze high salinity sea (over 30 parts per thousand) or open-ocean water samples which require higher dilution factors or contain (much) lower REE concentrations, respectively. Therefore, it was concluded that the direct determination of REEs is only possible from samples with moderate salinity such as estuarine or shallow-sea water. In the latter case, the longitudinal REE profiling assayed by ICP-MS allowed us to assume that the export of the contaminated material from land areas into estuaries and then to the sea by rivers may exert substantial contribution to the seawater pool of REEs.

Keywords Rare earth elements, analysis of seawater, ICP-MS, dilution, validation

\footnotetext{
${ }^{\dagger}$ To whom correspondence should be addressed.

E-mail: andrei.timerbaev@univie.ac.at
} 


\section{Introduction}

While by no means presenting the largest treat to the environment, rare earth elements (REEs) may constitute a potential risk to humans as their emission into seawater steadily increases with intensification of numerous industrial productions. ${ }^{1-3}$ Therefore, surveying seawater in terms of the level of REEs becomes a major concern to assess the state of marine ecosystems and anthropogenic impacts. ${ }^{4,5}$ There is another factor motivating an increasing attention to the determination of REEs in seawater. Chemical similarities render REEs useful as fingerprints in various geochemical processes along the water column, upon ocean circulation, etc. ${ }^{6-8}$

Extremely low concentrations of REEs, i.e. from low to sub-ng $\mathrm{L}^{-1}$ levels (for more detail, see Table S1; Supporting Information), and high salt content of seawater pose special requirements on analytical methodology in use. As a matter of fact, these challenges make inductively coupled plasma mass spectrometry (ICP-MS) the MVP in the field where the method shows an unparalleled performance. ${ }^{4,5}$ As follows from these excellent review papers, sample preparation is typically a mandatory step. It involves analyte preconcentration and matrix separation to transfer the enriched REEs into a solution free from the salt matrix, thus ensuring reliable ICP-MS measurements. Evidently, due to such treatment, commonly based on solid-phase extraction (see Table S1) utilizing the chelating resins variable in complexation chemistry, the analytical protocol turns out to be more complicated and prone to uncertainties. Last but not least, sample throughput suffers from time-consuming sample handling, which imposes constraint on method's applicability in country- or continent-wide ecological/geochemical projects with huge number of samples to deal with, including transport and storage. It should also be pointed out that however high sensitivity can be attained when a preconcentration step is accommodated, there seems to have been no ICPMS-based protocol for the quantification of seawater REEs established officially.

The direct determination of REEs using ICP-MS, if sufficiently sensitive, would obviously amend the above-mentioned limitations. However, examples of seawater analysis without intricate sample treatment are very few at the time of writing. Lawrence et al. applied a direct quadrupole-based technique to the analysis of a mid-salinity estuarine sample diluted from 17 to 1.8 parts per thousand (ppt). ${ }^{9}$ Chung and co-workers compared microconcentric and membrane-desolvation sample introduction systems in an attempt at enhancing the sensitivity of sector-field (SF) ICP-MS detection. ${ }^{10}$ However, their practical application to seawater was limited to analyzing - after a 100-fold dilution - a certified reference material (CRM) spiked with $1 \mu \mathrm{g} \mathrm{L}{ }^{-1}$ REEs. 
Recently, we have demonstrated that following substantial sample dilution a number of trace metals $\left(\mathrm{Pb}, \mathrm{Cd}, \mathrm{Th}, \mathrm{U}\right.$, etc.) are amenable to ICP-SFMS quantification in seawater. ${ }^{11}$ Such an approach takes advantage of both extremely high sensitivity of ICP-SFMS (by up to an order of magnitude over quadrupole instruments) and reduction of the matrix effect on instrumental sensitivity. With the objective of a large-scale monitoring of REEs, for which sample throughput is a basic prerequisite, we explore here how effective is the ICP-SFMS method to analyze seawater of varying salinity (from 3 to $30 \mathrm{ppt}$ ) after optimized sample dilution and validation including common analytical criteria and interlaboratory testing.

\section{Experimental}

\section{Instrumentation}

Analyses were performed on an Element-2 ICP-SFMS instrument (from Thermo Fisher Scientific, Waltham, MA, USA) with high resolution capacity, also available at Analytical Certification Testing Center Ltd., Moscow involved in interlaboratory studies. The instrument was used in low $(\mathrm{R}=300)$ resolution setting with the following operational parameters: plasma gas flow, $16 \mathrm{~L} \mathrm{~min}^{-1}$; auxiliary gas flow, $0.73 \mathrm{~L} \mathrm{~min}^{-1}$; analyzed sample flow, $0.78 \mathrm{~mL} \mathrm{~min}^{-1}$; RF power, $1200 \mathrm{~W}$; dwell time, $10 \mathrm{~ms}$. The REE isotopes measured in the experiments reported are listed in Table S2 (Supporting Information). The internal standard ${ }^{115} \mathrm{In}\left(1 \mu \mathrm{g} \mathrm{L}^{-1}\right)$, quite similar to REEs in mass and first ionization potential, was analyzed to account for any signal drift variation over time. Prior to measurements, the instrumental conditions (including torch position, sample gas flow rate, and lens settings) were optimized to achieve high, reproducible signal intensities and oxide/hydroxide production rates below $2 \%$. The isobaric interference of ${ }^{160} \mathrm{Dy}$ on ${ }^{160} \mathrm{Gd}$, as well as interferences from oxide and hydroxides of $\mathrm{Ba}$ on Eu and light REEs on heavy REEs, were quantified and subtracted in the usual way (see e.g. ref. 12). External calibration was performed at the beginning of each working day using a multi-element standard solution prepared as described below.

\section{Reagents and materials}

Stock solutions $\left(10 \mathrm{mg} \mathrm{L}^{-1}\right)$ used to prepare mixed standard solutions and the internal standard (High-Purity Standards, Charleston, SC, USA) were diluted appropriately with 3\% $\mathrm{HNO}_{3}$ prepared from ultrapure nitric acid (65\%, Merck, St. Louis, MO, USA) and ultrapure water. Seawater CRM (CASS-6, A33-11-02-CASS) was purchased from the National Research 
Council of Canada (Ottawa, ON). All plastic, quartz and glassware were soaked in $4 \mathrm{~mol} \mathrm{~L}^{-1}$ $\mathrm{HNO}_{3}$ for at least $24 \mathrm{~h}$ and rinsed repeatedly with ultrapure water before use.

\section{Sampling and sample preparation}

Sampling was done in July 2019 at different sites of the Kara Sea during a research oceanographic cruise aboard R.V. Academician Mstislav Keldysh. Geographical location of the sampling sites and sample salinities are detailed in Fig. S1 and Table S3 (Supporting Information), respectively. Samples were collected in duplicate (each sample 1 L) into Teflonlined lever-action Niskin bottles (General Oceanics, Miami, FL, USA). After filtration through acid pre-cleaned cellulose nitrate membrane filters (Whatman, $0.45 \mu \mathrm{m}$ ) under vacuum they were acidified (with $\mathrm{HNO}_{3}$ to $3 \%$ ) on board, stored at $4^{\circ} \mathrm{C}$ and later analyzed at the laboratory facilities, following dilution with $3 \% \mathrm{HNO}_{3}$ as described below.

\section{Data processing and validation}

All measurements were carried out in low resolution scanning mode that offered a much better sensitivity (by a factor over 50) and versatility than high resolution mode. Each sample was analyzed at least three times. The analytical parameters, evaluated to ensure the quality and comparability of results, included the limit of detection and quantification, measurement repeatability, intermediate (within-laboratory) precision, (between-laboratory) reproducibility, and accuracy.

\section{Results and discussion}

\section{Method precision and accuracy}

In order to explore the effect of sample dilution on method's performance, a series of dilution experiments were performed with the seawater sample of the highest salinity available (29.9 ppt). The data of Table 1 shows that with dilution from 10 to 100 times analytical precision (expressed here in terms of long-term variation) improves across each REE series, following elimination of the signal suppression due to the sea-matrix salinity. At the most extreme dilution, the SD values remain practically unchanged with larger deviations starting to appear only for a few elements. The results of within-day measurements were expectedly more repeatable, falling in the range from 3 to $5 \%(n=4)$ for all REEs analyzed. Analyses of the same sample and with the same dilution performed at the two laboratories 
(see above) revealed acceptable between-lab reproducibility, indicated by the RSD values lower than $14 \%(n=7)$. Variations appear to be attributed to different sample introduction systems rather than slight dissimilarities in instrumental parameters.

From the results in Table 1, the accuracy of the method was also assessed. It is evident that regardless of the dilution factor the trueness, calculated from deviations from the independently asserted values as a percentage recovery, amounted to more than $84 \%$ for all REEs, with the exception of least abundant Ho, Tm and Lu. It is important to mention that in contrast to the data of Lawrence et al., ${ }^{9}$ who employed quadrupole ICP-MS, the accuracy tests for Eu were fairly successful, e.g. $>90 \%$ with dilution 50 times. This observation supports the absence of a matrix effect due to the molecular ions ${ }^{135} \mathrm{Ba}^{16} \mathrm{O}^{+}$and ${ }^{137} \mathrm{Ba}^{16} \mathrm{O}^{+}$; barium is the minor metal component of seawater, ${ }^{13}$ exhibiting a three orders of magnitude concentration surplus over europium. In effect, a 50-fold dilution was established as superior for system performance regarding the accuracy of obtained results, as most of the REEs can be quantified within the acceptance range of 90-110\%. Poorer results for $\mathrm{Ho}, \mathrm{Tm}$ and $\mathrm{Lu}$, viz., measured concentrations lower than the recommended values, can be associated with contaminations from the single element standard used to prepare the internal standard solution.

To further control the reliability of the proposed method, the CRM of nearshore seawater was analyzed in separate experiments (Table 2). The results demonstrate a good concordance with respect to the information values (no certified values are offered for REEs in this CRM), varying from 3.0 to $8.6 \%$. The repeatability is better than $6 \%$ RSD for most of the REEs and only for $\mathrm{Tb}, \mathrm{Tm}$ and $\mathrm{Lu}$ was higher than $15 \%$. A careful reader would have noticed that REEs exist in the Kara Sea water at much higher concentrations compared to CASS6. While a questionable assumption without an in-depth investigation, seawater contamination might be due to a long-term use of Novaya Zemlya (consult the map in Fig. S1; Supporting Information) as a nuclear test site. ${ }^{14}$ In this context, a positive anomaly in the REE pattern relative to open-ocean concentrations should not be overlooked for cerium in view of its affiliations with the fission products of ${ }^{235} \mathrm{U}$ and ${ }^{239} \mathrm{Pu}$. Also notable are positive anomalies of heavy REEs whose large enrichment (3-4 times over natural abundancies) may be explained by the input of dissolved REEs from river water fluxes. ${ }^{14}$

\section{Sensitivity}

Among other factors, the LODs of the method are strongly affected by matrix composition. With this in mind, three sigma LODs were determined by taking into account the slope of the calibration curve plotted with sequential dilution of seawater from 10 to 200 
times (Table 3). The reached sensitivity thresholds varied from 0.04 to $0.4 \mathrm{ng} \mathrm{L}^{-1}$, being very similar to those reported using ICP-SFMS $\left(0.05-0.2 \mathrm{ng} \mathrm{L}^{-1}\right) .{ }^{10}$ Furthermore, our LODs occur to be not substantially worse than the LOD values obtained after analyte enrichment (see Table 3), especially for heavy REEs. However, from the summary of open-ocean REE concentrations presented in Table S1 (Supporting Information), it is obvious that the sensitivity of the proposed method is insufficient for quantification purposes given sample dilution (10 times and more). Still, the method was shown to be useful to reliably determine REEs in real seawater samples collected from contaminated offshore or nearshore areas (see also the analytical data given in the following section). The limits of quantification of the assay, defined as 10\%/slope at a standard 100-fold dilution, are listed in Table S4 (Supporting Information).

\section{Application - the longitudinal distribution of REEs}

Figure 1 shows the typical dependences of the REE concentrations on the distance from the estuary of the Ob River (see Fig. S1; Supporting Information) at which the sampling was done (the full set of REEs is presented in Table S5). The observed decrease in the metal contents indicates the effect of the freshwater carried down by river current which transfers organic matter and the associated metal fluxes. ${ }^{19,20}$ The fact that the river impact is still notable even at more than $250 \mathrm{~km}$ from the estuary should not misguide the reader. The Kara Sea is known for its vast shallow sections (average depth is less than $50 \mathrm{~m}$ across the sampling area) while the Ob River belongs to rivers with one of the largest discharges. Both hydrological elements favor the mass transfer effect.

Also, we compared the data for samples taken from the estuarine area (stations 6248 and 6251 with salinity below $10 \mathrm{ppt)}$ with the estuarine REE concentrations described in the literature. $^{21,22}$ Concentrations of most REEs proved to be within the range documented for estuarine and nearshore water.

The results of spatial distribution of REEs acquired by ICP-SFMS may be helpful to evaluate the ecological state of marine environment under scrutiny. Polluted at $50 \mathrm{~km}$ from the estuary, seawater tends to become less contaminated with distance in a pseudo linear fashion (see Fig. 1). However, even at $450 \mathrm{~km}$ from the coastline where the influx of the river into the sea fades out, the REE concentrations (though much lower than at the estuary) do not correspond to natural seawater levels. From comparison of the data for stations 6244 and 6224 , located at a distance of about $350 \mathrm{~km}$ from each other, it also appears that for whatever reason contamination remains valid. 


\section{Conclusions}

Direct ICP-SFMS analysis would obviously advance the means how the REE data for seawater are becoming available. Minimization of sample manipulation, such as that limited to simple dilution in the described procedure, improves the data quality by reducing blank contamination and sample throughput, let alone the cost of analysis. However, even with the latest generation of ICP-MS instruments the goal seems to be accessible only for samples with moderate salinity from the estuarine or nearshore areas or shallow seas (unless contamination is prominent). To give room for the direct analysis of open-ocean water, further instrumental progress is required, for instance, by improving sample introduction layout. An alternative approach is to optimize, validate and customize for routine use an ICP-MS-based procedure with matrix removal and REE preconcentration. In this regard, a compromise should be reached on the confronting issues of analyte enrichment factors and the time required to complete preconcentration. Pursuing both research avenues would present a step forward in terms of data availability and quality, an asset to reliably conducting environmental, oceanographic and geochemical projects.

\section{Acknowledgements}

Finding was provided by the Russian Ministry of Education and Science (state assignment No. 0137-2019-0020).

\section{Supporting Information}

Table S1, concentrations of REEs in open-ocean water; Table S2, analytical isotopes and possible polyatomic ion interferences; Fig. S1, sampling area; Table S3, sample salinity; Table 4, limits of quantification; Table S5, seawater REEs quantified at different distance from the coast. This material is available free of charge on the Web at http://www.jsac.or.jp/analsci/. 


\section{References}

1. K. Binnemans, P.T. Jones, T. Mueller, and L. Yurramendi, J. Sustain. Metallur., 2018, $3,126$.

2. W. Gwenzi, L. Mangori, C. Danha, N. Chaukura, N. Dunjana, and E. Sanganyado, Sci. Tot. Environ., 2018, 636, 299.

3. M. Edahbi, B. Plante. and M. Benzaazoua, J. Clean. Prod., 2019, 3, 1232.

4. A. Fisher and D. Kara, Anal. Chim. Acta, 2016, 935, 1.

5. I. Wysocka, Talanta, 2021, 221, 121636.

6. C. W. Noack, D. A. Dzombak, and A. K. Karamalidis, Environ. Sci. Technol., 2014, $48,4317$.

7. H. Song, W. J. Shin, J. S. Ryu, H. S. Shin, H. Chung, and K. S. Lee, Chemosphere, 2017, 172, 155.

8. K. C. Crocket, E. Hill, R. E. Abell, C. Johnson, S. F. Gary, T. Brand, and E. C. Hathorne, Front. Mar. Sci., 2018, 5, 1.

9. M. G. Lawrence, A. Greig, K. D. Collerson, and B. S. Kamber, Appl. Geochem., 2006, 21,839 .

10. C. H. Chung, I. Brenner, and C. F. You, Spectrochim. Acta B, 2009, 64, 849.

11. O. V. Kuznetsova and A. R. Timerbaev, At. Spectrosc., 2021, 42, 85.

12. M. P. Aliaga-Campuzano, J. P. Bernal, S. B. Briceno-Prieto, O. Perez-Arvizu, and E. Lounejeva, J. Anal. At. Spectrom., 2013, 28, 1102.

13. K. Fukushi, T. Hirokawa, and A. R. Timerbaev, J. Chromatogr. A, 2019, 1606, 360240.

14. Novaya Zemlya, https://en.wikipedia.org/wiki/Novaya_Zemlya (accessed March 2021).

15. Y. Zhu, A. Itoh, E. Fujimori, T. Umemura, and H. Haraguchi, J. Alloys Comp., 2006, $408-412,985$.

16. Y. Zhu, T. Umemura, H. Haraguchi, K. Inagaki, and K. Chiba, Talanta, 2009, 78, 891.

17. S. N. Willie and R. E. Sturgeon, Spectrochim. Acta B, 2001, 56, 1707.

18. N. Freslon, G. Bayon, D. Birot, C. Bollinger, and J. A. Barrat, Talanta, 2011, 85, 582.

19. O. V. Kuznetsova, Y. V. Bychkova, and A. R. Timerbaev, Anal. Lett., 2020, 53, 563.

20. O. V. Kuznetsova and A. R. Timerbaev, Anal. Lett., 2021, 54, 442. 
21. S. B. Adebayo, M. Cui, T. Hong, C. D. White, E. E. Martin, and K. H. Johannesson, Front. Marine Sci., 2018, 5, 166.

22. M. Casse, J.-C. Montero-Serrano, G. St-Onge, and A. Poirier, Marine Chem., 2019, $211,117$. 


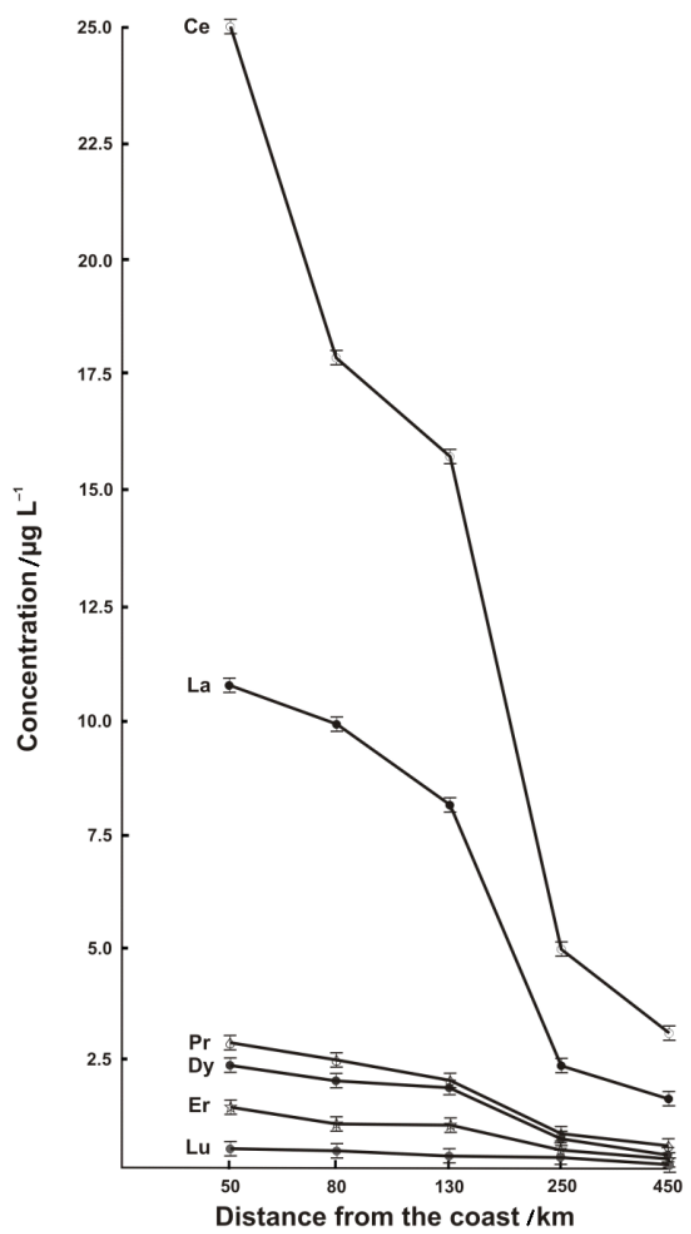

Fig. 1 Metal concentrations in seawater at different distance from the coast. 
Table 1

Intermediate precision and accuracy at different dilutions

\begin{tabular}{|c|c|c|c|c|c|c|c|c|c|c|c|c|}
\hline \multirow[t]{3}{*}{ REE } & \multicolumn{12}{|c|}{ Dilution factor } \\
\hline & \multicolumn{3}{|c|}{$1: 10$} & \multicolumn{3}{|c|}{$1: 50$} & \multicolumn{3}{|c|}{$1: 100$} & \multicolumn{3}{|c|}{$1: 200$} \\
\hline & $\begin{array}{c}\text { Mean } \\
/ \mu \mathrm{g} \mathrm{L}^{-1 a}\end{array}$ & SD & $\begin{array}{l}\text { Relative } \\
\text { error } / \%^{b}\end{array}$ & $\begin{array}{l}\text { Mean } \\
/ \mu \mathrm{g} \mathrm{L}^{-1}\end{array}$ & $\mathrm{SD}$ & $\begin{array}{l}\text { Relative } \\
\text { error/\% }\end{array}$ & $\begin{array}{l}\text { Mean } \\
/ \mu \mathrm{g} \mathrm{L}^{-1}\end{array}$ & SD & $\begin{array}{l}\text { Relative } \\
\text { error/\% }\end{array}$ & $\begin{array}{l}\text { Mean } \\
/ \mu \mathrm{g} \mathrm{L}^{-1}\end{array}$ & $\mathrm{SD}$ & $\begin{array}{l}\text { Relative } \\
\text { error } / \%\end{array}$ \\
\hline $\mathrm{La}$ & 6.86 & 0.31 & 16.6 & 6.48 & 0.21 & 8.4 & 6.75 & 0.04 & 14.8 & 6.89 & 0.04 & 17.1 \\
\hline $\mathrm{Ce}$ & 14.8 & 0.75 & 12.7 & 14.7 & 0.35 & 7.8 & 14.8 & 0.30 & 12.3 & 14.9 & 0.19 & 13.0 \\
\hline $\operatorname{Pr}$ & 1.77 & 0.11 & 11.5 & 1.74 & 0.06 & 9.0 & 1.82 & 0.03 & 14.6 & 1.84 & 0.21 & 15.7 \\
\hline $\mathrm{Nd}$ & 6.67 & 0.34 & 1.1 & 6.53 & 0.18 & 3.2 & 6.99 & 0.08 & 3.6 & 6.91 & 0.13 & 2.4 \\
\hline $\mathrm{Sm}$ & 1.36 & 0.15 & 7.3 & 1.36 & 0.06 & 7.3 & 1.41 & 0.01 & 4.1 & 1.39 & 0.03 & 5.4 \\
\hline $\mathrm{Eu}$ & 0.34 & 0.03 & 8.3 & 0.34 & 0.01 & 8.3 & 0.35 & 0.01 & 11.3 & 0.37 & 0.03 & 18.0 \\
\hline $\mathrm{Gd}$ & 1.36 & 0.15 & 3.9 & 1.43 & 0.05 & 1.1 & 1.36 & 0.03 & 3.7 & 1.36 & 0.03 & 3.7 \\
\hline $\mathrm{Tb}$ & 0.22 & 0.02 & 13.8 & 0.22 & 0.01 & 9.2 & 0.23 & 0.01 & 16.6 & 0.25 & 0.01 & 28.7 \\
\hline Dy & 1.07 & 0.09 & 2.0 & 1.05 & 0.05 & 0.1 & 1.09 & 0.02 & 3.6 & 0.99 & 0.04 & 5.3 \\
\hline Ho & 0.20 & 0.03 & 31.8 & 0.19 & 0.01 & 35.2 & 0.20 & 0.01 & 32.0 & 0.20 & 0.01 & 31.8 \\
\hline $\mathrm{Er}$ & 0.55 & 0.05 & 5.7 & 0.54 & 0.01 & 3.7 & 0.57 & 0.01 & 10.3 & 0.58 & 0.01 & 12.1 \\
\hline $\mathrm{Tm}$ & 0.062 & 0.014 & 43.2 & 0.070 & 0.003 & 68.6 & 0.070 & 0.006 & 64.7 & 0.071 & 0.006 & 64.7 \\
\hline $\mathrm{Yb}$ & 0.41 & 0.04 & 2.6 & 0.40 & 0.01 & 0.1 & 0.42 & 0.02 & 4.4 & 0.42 & 0.02 & 5.6 \\
\hline $\mathrm{Lu}$ & 0.060 & 0.005 & 43.8 & 0.062 & 0.002 & 48.6 & 0.067 & 0.002 & 59.4 & 0.068 & 0.003 & 62.8 \\
\hline
\end{tabular}

${ }^{\bar{a}}$ Laboratory average of three-day measurements over 4-week period. ${ }^{b}$ Compared with the specified values (provided by Analytical Certification Testing Center). 
Table 2 Accuracy and precision for analysis of CASS- $6^{a}$

\begin{tabular}{llll}
\hline REE & $\begin{array}{l}\text { Information } \\
\text { value/ng L }\end{array}$ & $\begin{array}{l}\text { Measured value } \\
\text { /ng L }\end{array}$ & $\begin{array}{l}\text { Relative } \\
\text { error/\% }\end{array}$ \\
\hline $\mathrm{La}$ & 7.0 & $7.6 \pm 0.3$ & 8.6 \\
$\mathrm{Ce}$ & 5.0 & $4.8 \pm 0.2$ & 4.0 \\
$\mathrm{Pr}$ & 2.0 & $2.1 \pm 0.1$ & 5.0 \\
$\mathrm{Nd}$ & 4.0 & $3.9 \pm 0.2$ & 2.5 \\
$\mathrm{Sm}$ & 4.0 & $4.3 \pm 0.2$ & 7.5 \\
$\mathrm{Eu}$ & 2.0 & $1.9 \pm 0.1$ & 7.5 \\
$\mathrm{Gd}$ & 2.0 & $2.1 \pm 0.1$ & 3.0 \\
$\mathrm{~Tb}$ & 0.3 & $0.28 \pm 0.05$ & 6.7 \\
$\mathrm{Dy}$ & 1.4 & $1.3 \pm 0.1$ & 7.1 \\
$\mathrm{Ho}$ & 2.0 & $2.1 \pm 0.1$ & 5.0 \\
$\mathrm{Er}$ & 1.4 & $1.5 \pm 0.1$ & 6.4 \\
$\mathrm{Tm}$ & 0.2 & $0.19 \pm 0.07$ & 5.0 \\
$\mathrm{Yb}$ & 1.6 & $1.7 \pm 0.1$ & 6.2 \\
$\mathrm{Lu}$ & 0.4 & $0.37 \pm 0.08$ & 7.5 \\
\hline $\mathrm{Dilut}$ & & &
\end{tabular}

${ }^{a}$ Dilution, $1: 10 .{ }^{b} n=3$. 
Table 3 Comparison of detection limits (in $\mathrm{ng} \mathrm{L}^{-1}$ )

\begin{tabular}{lccccc}
\hline REE & \multicolumn{5}{c}{ Literature data $^{a}$} \\
& 15 & 16 & 17 & 18 & Our \\
\hline $\mathrm{La}$ & 0.10 & 0.040 & 0.05 & 0.012 & 0.22 \\
$\mathrm{Ce}$ & 0.10 & 0.090 & 0.04 & 0.020 & 0.38 \\
$\mathrm{Pr}$ & 0.07 & 0.011 & 0.04 & 0.007 & 0.17 \\
$\mathrm{Nd}$ & 0.30 & 0.080 & 0.29 & 0.029 & 0.31 \\
$\mathrm{Sm}$ & 0.50 & 0.017 & 0.08 & 0.015 & 0.13 \\
$\mathrm{Eu}$ & 0.10 & 0.007 & 0.03 & 0.006 & 0.09 \\
$\mathrm{Gd}$ & 0.40 & 0.014 & 0.08 & 0.016 & 0.25 \\
$\mathrm{~Tb}$ & 0.08 & 0.008 & 0.02 & 0.004 & 0.06 \\
$\mathrm{Dy}$ & 0.30 & 0.013 & 0.12 & 0.005 & 0.14 \\
$\mathrm{Ho}$ & 0.07 & 0.005 & 0.02 & 0.005 & 0.09 \\
$\mathrm{Er}$ & 0.20 & 0.008 & 0.06 & 0.006 & 0.11 \\
$\mathrm{Tm}$ & 0.08 & 0.005 & 0.02 & - & 0.04 \\
$\mathrm{Yb}$ & 0.30 & 0.009 & 0.16 & 0.008 & 0.10 \\
$\mathrm{Lu}$ & 0.06 & 0.009 & 0.02 & 0.001 & 0.05 \\
\hline
\end{tabular}

${ }^{a}$ With ICP-MS after preconcentration. 